Pacific Journal of Mathematic 


\section{THE RADICAL OF A REFLEXIVE OPERATOR ALGEBRA}

\section{Alan Hopenwasser}

The radical of a reflexive operator algebra $\mathfrak{U}$ whose lattice of invariant subspaces $\mathcal{L}$ is commutative is related to the space of lattice homomorphisms of $\mathfrak{Z}$ onto $\{0,1\}$. To each such homomorphism $\phi$ is associated a closed, two-sided ideal $\mathfrak{A}_{\phi}$ contained in $\mathfrak{U}$. The intersection of the $\mathfrak{U}_{\phi}$ is contained in the radical; it is conjectured that equality always holds. The conjecture is proven for a variety of special cases: countable direct sums of nest algebras; finite direct sums of algebras which satisfy the conjecture; algebras whose lattice of invariant subspaces is finite; algebras whose lattice of invariant subspaces is isomorphic to the lattice of nonincreasing sequences with values in $N \cup\{\infty\}$.

1. Introduction. This paper studies the radical of a certain class of non-self-adjoint operator algebras. Given an algebra $\mathfrak{A}$ and a lattice $\mathbb{R}$ of orthogonal projections acting on a separable Hilbert space $\mathfrak{S}$, we use the standard notations, \&at $\mathfrak{A}$ and $\mathfrak{A l g} \mathfrak{L}$, to denote, respectively, the lattice of all projections invariant under $\mathfrak{A}$ and the algebra of all (bounded) operators which leave invariant each projection of $\mathfrak{R}$. $\mathfrak{A}$ and $\mathbb{Z}$ are said to be reflexive if $\mathfrak{A}=\mathfrak{A l g}$ Rat $\mathfrak{A}$ and $\mathfrak{L}=\mathbb{R} a t \mathfrak{U l g} \&$, respectively. The algebras which we study are reflexive algebras which contain a maximal abelian self-adjoint algebra (m.a.s.a.).

A commutative subspace lattice is a lattice of pairwise commuting, orthogonal projections on $\mathfrak{F}$ which contains 0 and 1 and which is closed in the strong operator topology. It follows automatically that a commutative subspace lattice is a complete lattice. If $\mathfrak{X}$ is an operator algebra containing a m.a.s.a., then Rat $\mathfrak{X}$ is a commutative subspace lattice. Every commutative subspace lattice, $\mathfrak{L}$, is reflexive ([1], p. 468), and $\mathfrak{A l g} \mathfrak{Q}$ is a reflexive algebra which contains a m.a.s.a. Henceforth, all lattices of projections in this paper will be commutative subspace lattices and all algebras will be reflexive algebras which contain a m.a.s.a. An incisive study of these lattices and algebras by Arveson is found in [1].

At least in certain special cases, the radical of a reflexive algebra, $\mathfrak{A}$, containing a m.a.s.a. can be described in terms of the set of lattice homomorphisms from $\mathfrak{L}=\mathfrak{L a t} \mathfrak{A}$ onto $\{0,1\}$. To each such homomorphism $\phi$ we shall associate a closed two-sided ideal $\mathfrak{A}_{\phi}$ in $\mathfrak{A}$. The radical, $\Re$, of $\mathfrak{A}$ is equal to the intersection of these ideals. It appears reasonable to conjecture that this equality holds for all 
algebras in this class. The conjecture serves as a test problem for our understanding of the algebraic structure of these algebras.

2. General results. For the following, fix $\&$ as a commutative subspace lattice and $\mathfrak{A}=\mathfrak{N} \mathfrak{l g}(\Omega)$. Let 2 denote the trivial lattice $\{0,1\}$ with the usual lattice structure. Let $X=X(\Omega)$ be the set of lattice homomorphisms from $\&$ onto 2 . Observe that $X$ is a subset of $2^{\varepsilon}=\Pi_{8} 2$, the set of functions defined on $\&$ with values in 2 . Put the discrete topology on 2 , the product topology on $2^{3}$, and the relative topology on $X$. We claim that $X$ is a closed subset of $2^{8}$. Indeed, if $\phi_{\nu} \in X$ and $\phi_{\nu} \rightarrow \phi$, where $\phi \in 2^{R}$, then for each $E \in \mathbb{R}$, there is a $\nu_{0}$, such that $\nu \geqq \nu_{0}$ implies $\phi_{\nu}(E)=\phi(E)$. With $E$ and $F$ arbitrary in 2 , choose $\nu$ sufficiently large that $\phi_{\nu}(E)=\phi(E)$ and $\phi_{\nu}(F)=$ $\phi(F)$. Since $\phi_{\nu}$ is a lattice homomorphism, we obtain $\phi(E \wedge F)=$ $\phi(E) \wedge \phi(F)$ and $\phi(E \vee F)=\phi(E) \vee \phi(F)$. Thus $\phi$ is a lattice homomorphism, and so $\phi \in X$. Since $2^{\mathfrak{2}}$ is a compact Hausdorff space and $X$ is closed, we see that $X$ also is a compact Hausdorff space.

If $\phi \in X$, let $\operatorname{ker} \phi=\{E \mid \phi(E)=0\}$ and coker $\phi=\{E \mid \phi(E)=1\}$. It is immediate that $\operatorname{ker} \phi$ is an ideal and coker $\phi$ is a co-ideal.

(An ideal is a subset $\&$ of $\&$ which satisfies the properties:

(a) $E_{1} \in$ SS, $E_{2} \in$ SS $\rightarrow E_{1} \vee E_{2} \in$ BS,

(b) $E \in \mathbb{B}, G \in \mathbb{R}, G \leqq E \rightarrow G \in \mathbb{B}$.

A co-ideal is a subset $\Im$ which satisfies the dual properties:

(a') $F_{1} \in \mathfrak{\Im}, F_{2} \in \mathfrak{\Im} \rightarrow F_{1} \wedge F_{2} \in \mathfrak{\Im}$

(b') $F \in \mathfrak{\Im}, G \in \mathbb{R}, F \leqq G \rightarrow G \in \Im$.

An ideal is prime if its complement is a co-ideal. The prime ideals of $\mathbb{Z}$ are precisely the kernels of the lattice homomorphisms onto 2 . (See $[2]$, p. 28.)

Definition. A family, $\mathfrak{F}$, of non-zero orthogonal projections in $\mathfrak{A}$ is called a basic family provided:

(i) Each $P \in \mathscr{F}$ is of the form $P=F-E$, where $F, E \in \mathbb{Q}$ and $E<F$,

(ii) $\mathfrak{F}$ satisfies the finite intersection property,

(iii) $\mathfrak{F}$ is maximal with respect to properties (i) and (ii).

REMarks. Condition (i) guarantees that $\mathfrak{F}$ is contained in the (abelian) algebra generated by $\mathbb{R}$; hence, condition (ii) simply means that, if $P_{1}, \cdots, P_{n} \in \mathfrak{F}$, then $P=P_{1} P_{2}, \cdots, P_{n}$ is a nonzero projection. Condition (iii) ensures that $P \in \mathfrak{F}$.

Lemma 1. The set $X$ of lattice homomorphisms of $\mathbb{R}$ onto 2 is in a natural one-to-one correspondence with the set of basic families of projections in $\mathfrak{A}$. Explicitly, to each $\phi$ in $X$ associate the basic 
family $\mathfrak{F}=\{F-E \mid E<F, E \in \operatorname{ker} \phi$, and $F \in$ coker $\phi\}$. The inverse is given by associating to each basic family $\mathfrak{F}$ the unique lattice homomorphism $\phi$ in $X$ whose co-kernel is the prime co-ideal $\mathfrak{F} \cap \mathbb{R}$.

Proof. Assume $\phi \in X$ and let $\widetilde{F}=\{F-E \mid E<F, E \in \operatorname{ker} \phi$, and $F \in$ coker $\phi$ \}. It is immediate that $\widetilde{F}$ satisfies condition (i). To show condition (ii) is satisfied, it suffices to show that $P, Q \in \mathfrak{F}$ implies $P Q \in \mathfrak{F}$. If $P=F_{1}-E_{1}$ and $Q=F_{2}-E_{2}$, with $F_{1}, F_{2} \in$ coker $\phi$ and $E_{1}, E_{2} \in \operatorname{ker} \phi$, then $P Q=F_{1}\left(1-E_{1}\right) F_{2}\left(1-E_{2}\right)=F_{1} F_{2}\left(1-E_{1}-E_{2}+\right.$ $\left.E_{1} E_{2}\right)=F_{1} F_{2}\left(1-E_{1} \vee E_{2}\right)=F_{1} \wedge F_{2}-\left[\left(E_{1} \vee E_{2}\right) \wedge\left(F_{1} \wedge F_{2}\right)\right]$. Now, $\phi\left(F_{1} \wedge F_{2}\right)=\phi\left(F_{1}\right) \wedge \phi\left(F_{2}\right)=1$ and $\phi\left(\left(E_{1} \vee E_{2}\right) \wedge\left(F_{1} \wedge F_{2}\right) \leqq \phi\left(E_{1} \vee E_{2}\right)=\right.$ $\phi\left(E_{1}\right) \vee \phi\left(E_{2}\right)=0$, hence $P Q \in \mathfrak{F}$. To verify condition (iii), suppose $\mathbb{B}$ is a family of projections in $\mathscr{A}$ which contains $\mathfrak{F}$ and satisfies conditions (i) and (ii). Let $P$ be an arbitrary element of $\$$. Then $P=$ $F-E$, for some $E, F \in \mathbb{\&}$ with $E<F$. If $\phi(F)=0$, then $1-F \in \mathfrak{F} \subset$ (B) and (S) does not satisfy (ii); hence $\phi(F)=1$. Similarly, if $\phi(E)=1$, then $E=E-0 \in \mathfrak{\Im} \subset \mathbb{B S}$ and again (S) cannot satisy condition (ii); so $\phi(E)=0$. But $E \in \operatorname{ker} \phi$ and $F \in$ coker $\phi$ imply that $P=F-E \in \mathfrak{F}$. Thus $\mathbb{B}=\widetilde{F}$ and $\mathfrak{F}$ is a basic family.

Now assume $\widetilde{\Im}$ is a basic family of projections in 2 . If $E$ is an arbitrary projection in $\mathcal{L}$, then either $E$ intersects each projection of $\widetilde{\mho}$ or $1-E$ intersects each projection of $\widetilde{\Im}$. For, if there exist $P, Q \in \widetilde{F}$ with both $P \wedge E=0$ and $Q \wedge(1-E)=0$, then $P \leqq 1-E$ and $Q \leqq E$, whence $P Q=0$, a contradiction. If $E$ intersects each projection of $\widetilde{F}$, then $\widetilde{\mho} \cup\{E\}$ satisfies (i) and (ii); likewise, if $1-E$ intersects each projection of $\mathfrak{F}$, then $\mathfrak{F} \cup\{1-E\}$ satisfies (i) and (ii). Since $\widetilde{F}$ is maximal, we may conclude that, for each $E \in \mathbb{R}$, either $E \in \mathfrak{F}$ or $1-E \in \mathfrak{F}$.

Now let $\mathbb{S}=\{F \in \mathbb{R} \mid F \in \mathfrak{F}\}=\mathfrak{F} \cap \mathbb{Q}$ and $\mathfrak{\Im}=\{E \in \mathbb{Q} \mid 1-E \in \mathfrak{F}\}$. It is straightforward to check that $\mathscr{B}$ is a co-ideal and $\mathfrak{\Im}$ is an ideal. The paragraph above shows that $\mathfrak{\Im}$ is prime, and this determines the lattice homomorphism $\phi$ associated with $\mathfrak{F}$.

It will be convenient in the sequel to call a projection $P$ of the form $P=F-E$, where $E, F \in \mathbb{Q}$ and $E<F$, an interval projection from \&. If $\phi$ is an element of $X$ such that $\phi(F)=1$ and $\phi(E)=0$, we say $P$ is a test-interval for $\phi$.

In the case in which $\mathcal{L}$ is totally ordered (and so $\mathfrak{U}$ is a nest algebra), it is easy to describe the elements of $X$. For each $E \neq I$ in \&, let $\phi_{E}^{+}$be defined by the formula,

$$
\phi_{E}^{+}(F)=\left\{\begin{array}{lll}
1 & \text { if } \quad F>E \\
0 & \text { if } \quad F \leqq E
\end{array}\right.
$$


For each $E \neq 0$ in $\&$, let $\phi_{\vec{E}}$ be defined by the formula,

$$
\phi_{\bar{E}}^{-}(F)=\left\{\begin{array}{lll}
1 & \text { if } \quad F \geqq E \\
0 & \text { if } \quad F<E .
\end{array}\right.
$$

It is immediate that the $\phi_{E}^{+}, \phi_{E}^{-}$all lie in $X$ and that, if $E$ is an immediate predecessor of $G$, then $\phi_{E}^{+}=\phi_{G}$. Further, every lattice homomorphism $\phi$ in $X$ arises in this fashion. Indeed, since $\&$ is totally ordered, each projection in the co-kernel of $\phi$ dominates each projection in the kernel of $\phi$. Let $E=\Lambda\{F \mid \phi(F)=1\}$ and $G=$ V $\{F \mid \phi(F)=0\}$. If $E \neq G$, then $G$ is the immediate predecessor of $E$ and $\phi=\phi_{E}^{-}=\phi_{G}^{+}$. If $G=E$, then $\phi$ is $\phi_{E}^{+}$or $\phi_{E}$ according as $\phi(E)=$ 0 or $\phi(E)=1$, respectively. We can define a total ordering on $X$ as follows: if $E<G$ then we say $\phi_{E}^{ \pm}<\phi_{G}^{ \pm}$(except when $\phi_{E}^{+}=\phi_{G}^{-}$); we define $\phi_{E^{-}}<\phi_{E^{+}}$for all $E \neq 0, I$. This ordering induces a topology on $X$, which coincides with the topology defined above.

In the particular case in which $\&$ is order isomorphic to the unit interval, $I=[0,1]$, we may realize the topological space $X$ in an amusing way. Let the cartesian product $I \times I$ be provided with the lexicographic order: $(a, b)<(c, d)$ if $a<c$ or if $a=c$ and $b<d$. Let $I \times I$ have the order topology induced by this order and let $Y$ be the subset, $\{(a, b) \mid$ either $b=0$ and $a \neq 0$, or $b=1$ and $a \neq 1\}$, provided with the relative topology. It is easy to see that $Y$ is homeomorphic to $X$.

We return to the general case in which $\mathcal{L}$ is any commutative subspace lattice (on separable Hilbert space), $\mathfrak{X}$ is the reflexive algebra, $\mathfrak{A l g}(\&)$, and $X$ is the space of lattice homomorphisms of $\mathbb{L}$ onto 2 .

Definitions. For each $\phi \in X$ and $T \in A$, defined

$$
N_{\phi}(T)=\inf \{\|P T P\| \mid P \text { is a test interval for } \phi\} \text {. }
$$

For each $\phi \in X$, define $\mathfrak{N}_{\phi}=\left\{T \in \mathfrak{X} \mid N_{\phi}(T)=0\right\}$.

Lemma 2. $N_{\phi}$ is a continuous mapping of $\mathfrak{X}$ onto $R^{+}$and it is a semi-norm.

Proof. To prove continuity, suppose $T_{\nu} \rightarrow T$. Let $\varepsilon>0$. Choose $\nu_{0}$ such that $\nu \geqq \nu_{0}$ implies $\left\|T_{\nu}-T\right\|<\varepsilon$. Then, if $P$ is any test interval for $\phi,\left\|P T_{\nu} P-P T P\right\|<\varepsilon$. Hence, $\|P T P\|-\varepsilon<\left\|P T_{\nu} P\right\|<$ $\|P T P\|+\varepsilon$. It then follows that $N_{\phi}(T)-\varepsilon<N_{\phi}\left(T_{\nu}\right)<N_{\phi}(T)+\varepsilon$. Thus $N_{\phi}\left(T_{\nu}\right) \rightarrow N_{\phi}(T)$.

It is immediate that $N_{\phi}(\lambda T)=|\lambda| N_{\phi}(T)$. To prove the subadditivity of $N_{\phi}$, suppose $S$ and $T$ belong to $\mathscr{A}$ and let $P_{1}$ and $P_{2}$ be test intervals for $\phi$ such that $\left\|P_{1} S P_{1}\right\|<N_{\phi}(S)+\varepsilon / 2$ and $\left\|P_{2} T P_{2}\right\|<$ 
$N_{\phi}(T)+\varepsilon / 2$, where $\varepsilon>0$. Then $P=P_{1} P_{2} \neq 0$ is a test interval for $\phi$ and $\|P(S+T) P\| \leqq\|P S P\|+\|P T P\| \leqq\left\|P_{1} S P_{1}\right\|+\left\|P_{2} T P_{2}\right\| \leqq N_{\phi}(S)+$ $N_{\phi}(T)+\varepsilon$. Since $\varepsilon$ is arbitrary, we obtain $N_{\phi}(S+T) \leqq N_{\phi}(S)+$ $N_{\phi}(T)$. Thus $N_{\phi}$ is a semi-norm.

Lemma 3. $\mathfrak{A}_{\phi}$ is a closed two-sided ideal in $\mathfrak{A}$.

Proof. $\mathfrak{A}_{\phi}$ is closed since $N_{\phi}$ is continuous and $\mathfrak{A}_{\phi}$ is a linear subspace of $\mathfrak{A}$ since $N_{\phi}$ is a semi-norm. Suppose $T \in \mathfrak{A}_{\phi}$ and $S$ is any element of 2 . If $P=F-E$ is a test interval for $\phi$ then PSTP $=$ PSPTP. (This follows from the facts $(I-F) S=(I-F) S(I-F)$ and $T E=E T E$.) Hence $\|P S T P\| \leqq\|S\|\|P T P\|$, which implies that $N_{\phi}(S T)=0$. In the same way it can be shown that $N_{\phi}(T S)=0$; thus $\mathfrak{A}_{\phi}$ is a two-sided ideal.

REMARK. The mapping $\phi \rightarrow N_{\phi}(T)$ is not continuous. It is true that if $\dot{\phi}_{\nu} \rightarrow \dot{\phi}$ in $X$, then $\lim \sup N_{\phi_{\nu}}(T) \leqq N_{\phi}(T)$; but strict inequality may occur. Indeed, given $\varepsilon>0$, let $P=F-E$ be a test interval for $\phi$ such that $\|P T P\| \leqq N_{\phi}(T)+\varepsilon$. Since $\phi_{\nu} \rightarrow \phi$, there exists a $\nu_{0}$ such that $\nu \geqq \nu_{0}$ implies $\phi_{\nu}(F)=\phi(F)=1$ and $\phi_{\nu}(E)=\phi(E)=0$. Hence, $P$ is a test interval for $\phi_{\nu}$, and $N_{\phi_{\nu}}(T) \leqq\|P T P\| \leqq N_{\phi}(T)+\varepsilon$. As $\varepsilon$ is arbitrary, $\lim \sup N_{\phi_{\nu}}(T) \leqq N_{\phi}(T)$.

For an example of strict inequality, let $\mathfrak{S}=L^{2}[0,1]$ (with Lebesgue measure) and let $E_{t}$ be the projection corresponding to the set $[0, t]$, for each $t \in[0,1]$. Let $\mathfrak{L}=\left\{E_{t} \mid t \in[0,1]\right\}$ and $\mathfrak{A}=\mathfrak{A} \mathfrak{I g} \mathfrak{L}$. Let $P$ be the projection corresponding to the union of all the intervals of the form $\left(2^{-n-1}, 2^{-n}\right)$ with $n$ even. For each odd $n$, let $t_{n}$ be the mid-point of the interval $\left(2^{-n-1}, 2^{-n}\right)$, and let $\phi_{n}$ be either of the lattice homomorphisms associated with $E_{t_{n}}$. (It is irrelevant which is chosen.) It is easy to see that $\phi_{n} \rightarrow \phi_{0}^{+}$in $X$ (as $n \rightarrow \infty$ with $n$ odd), that $N_{\phi_{n}}(P)=0$, for all odd $n$, and that $N_{\phi_{0}^{+}}(P)=1$.

We now consider the relation between the ideals $\mathfrak{A}_{\phi}$ and the radical of the reflexive algebra $\mathfrak{A}$. Recall ([3], Chapter 2, §3) that if $\mathfrak{R}$ is the radical of $\mathfrak{A}$ then

$\Re=\bigcap\{\operatorname{ker} \pi \mid \pi$ is a continuous topologically irreducible representation on a Banach space

$=\{T \mid S T$ is quasi-nilpotent, for all $S \in \mathfrak{A}\}$

$=\{T \mid T S$ is quasi-nilpotent, for all $S \in \mathfrak{U}\}$.

Proposition 4. $\bigcap_{\phi \in X} \mathfrak{A}_{\phi} \subseteq \Re$.

Proof. Let $T \in \bigcap_{\phi \in X} \mathfrak{A}_{\phi}$. Let $\pi$ be any continuous irreducible representation of $\mathfrak{A}$ acting on a Banach space $Y$. Since any $E$ in $\mathfrak{L}$ 
is idempotent, $\pi(E)$ is a projection. Since $S E=E S E$, for all $S \in \mathfrak{A}$, $\pi(S) \pi(E)=\pi(E) \pi(S) \pi(E)$, for all $S \in \mathfrak{A}$, and so the range of $\pi(E)$ is left invariant under the representation $\pi$. Thus $\pi(E)=0$ or $\pi(E)=I$, for each $E \in \mathbb{R}$. Let $\phi$ be the restriction of $\pi$ to \&. $\phi$ is thus identified with an element of $X$. Given $\varepsilon>0$, choose a test interval $F-E$ such that $\|(F-E) T(F-E)\|<\varepsilon$. Then, since $\pi(F-E)=\pi(F)-\pi(E)=I-0=I$, we have

$$
\|\pi(T)\|=\|\pi((F-E) T(F-E))\| \leqq\|\pi\| \varepsilon .
$$

As $\varepsilon$ is arbitrary, $\|\pi(T)\|=0$ and $T \in \operatorname{ker} \pi$. This is true for all continuous topologically irreducible representations, hence $T \in \Re$.

REMARK. If $\mathfrak{Q}$ is complemented then $\mathfrak{A}$ is a von Neumann algebra, hence $\Re=0$. From this it follows that $\bigcap_{\phi \in X} \mathfrak{A}_{\dot{\phi}}=\Re$. At the other extreme, if $\mathfrak{Z}$ is totally ordered (and so $\mathfrak{A}$ is a nest algebra), then again $\bigcap_{\phi \in X} \mathfrak{A}_{\phi}=\Re$. This result is due to Ringrose [4]; for the convenience of the reader a sketch of a somewhat simplified proof of this theorem will be given later.

Let us agree to say that an algebra $\mathscr{O}$ satisfies the radical condition if $\bigcap_{\phi \in X} \mathfrak{A}_{\phi}=\mathfrak{R}$. We conjecture that any reflexive algebra which contains a m.a.s.a. satisfies the radical condition. We shall show in this paper that any algebra with a finite (commutative) subspace lattice satisfies the radical condition; that the radical condition is satisfied by a finite direct sum of algebras, each of which satisfies the radical condition; and that an arbitrary (countable) direct sum of nest algebras satisfies the radical condition. We shall also show that the radical condition is satisfied by any algebra whose lattice of invariant projections is isomorphic to what may be described as the tensor product of the lattice $N \cup\{\infty\}$ with itself.

As a result of Proposition 4, the problem consists of proving that $\mathfrak{R} \subseteq \mathfrak{A}_{\phi}$, for each $\phi \in X(\Omega)$. Where difficulties arise, they are caused primarily by the lack of an explicit description of the $\phi \in X$.

We begin with a crude classification of the lattice homomorphisms of $X$. Fix $\dot{\phi} \in X$ and denote:

$$
\begin{aligned}
& E_{-}=\bigvee\{F \in \mathfrak{L} \mid \phi(F)=0\} \\
& E_{+}=\Lambda\{F \in \mathfrak{S} \mid \phi(F)=1\}
\end{aligned}
$$

Each of the following possibilities may occur:

(1) $E_{-}=E_{+}$.

(2) $E_{-}<E_{+}$.

(3) $E_{+}<E_{-}$.

(4) $E_{-}$and $E_{+}$are not comparable.

(a) $\phi\left(E_{-}\right)=0$ and $\phi\left(E_{+}\right)=0$. 
(b) $\phi\left(E_{-}\right)=0$ and $\phi\left(E_{+}\right)=1$.

(c) $\phi\left(E_{-}\right)=1$ and $\phi\left(E_{+}\right)=0$.

(d) $\phi\left(E_{-}\right)=1$ and $\phi\left(E_{+}\right)=1$.

Proposition 5. The only possible combinations from the two lists are the following: $1 \mathrm{a}, 1 \mathrm{~d}, 2 \mathrm{~b}, 3 \mathrm{a}, 3 \mathrm{c}, 3 \mathrm{~d}$, and $4 \mathrm{~b}$.

Proof. It is immediate that 1 is incompatible with $\mathrm{b}$ or $\mathrm{c}$, and that $2 \mathrm{c}$ and $3 \mathrm{~b}$ are impossible. If $\phi\left(E_{+}\right)=0$ then, by the definition of $E_{-}$, we have $E_{+} \leqq E_{-}$. This eliminates $2 \mathrm{a}, 4 \mathrm{a}$, and $4 \mathrm{c}$. If $\phi\left(E_{-}\right)=$ 1 , then $E_{+} \leqq E_{-}$, eliminating $2 \mathrm{~d}$ and $4 \mathrm{~d}$. Only the specified possibilities remain.

Examples of homomorphisms of types $1 \mathrm{a}, 1 \mathrm{~d}$, and $2 \mathrm{~b}$ can be obtained from nest algebras. Assume $\&$ is totally ordered. If $F$ is an element of $\mathbb{Z}$ with no immediate successor, then $\phi_{F}^{+}$is of type 1 . If, on the other hand, $F$ has no immediate predecessor, then $\phi_{\vec{F}}$ is of type 1d. Finally, if $G$ is an immediate predecessor to $F$, then $\dot{\phi}_{G}^{+}=\phi_{\bar{F}}$ is of type $2 \mathrm{~b}$. Examples of types $3 \mathrm{a}, 3 \mathrm{c}, 3 \mathrm{~d}$, and $4 \mathrm{~b}$ will be given later.

Definition. An interval projection $P$ from $\mathbb{R}$ is called an atom if, for any $E \in \mathbb{R}$, either $P \leqq E$ or $P E=0$.

REMARK. If $P$ is an atom then it is evident that $\{E \in \mathbb{R} \mid P E=0\}$ is an ideal in $\mathscr{R}$, while its complement, $\{E \in \mathfrak{L} \mid P \leqq E\}$ is a co-ideal. Hence $P$ determines an element $\phi$ of $X$, where $\phi$ is defined by $\phi(E)=1$ if and only if $P \leqq E$. Since $P$ is an interval projection, $P$ is in the basic family associated with $\phi$ and it is clear that $P$ is a sub-projection of each projection in that basic family. Hence $N_{\phi}(T)=\|P T P\|$, for all $T \in \mathfrak{A}$. The fact that $P$ is an interval also implies that the mapping $\pi(T)=P T P$ is a representation. This representation is clearly continuous and, when $P$ is an atom, it is also irreducible. (Any element of $\mathfrak{B}\left(P S_{\mathcal{C}}\right)$ can be extended to an operator $S$ on $\mathfrak{S}$ such that $S=P S P$. Since every projection $E$ in $\mathbb{R}$ either contains $P$ or is orthogonal to $P$, it follows that $S$ leaves each such projection invariant. Thus $S \in \mathfrak{A}$ and $\pi(\mathfrak{A})=\mathfrak{B}(P \mathfrak{K})$.) So we see that $\mathfrak{U}_{\phi}=\operatorname{ker} \pi$ and from this it is clear that $\Re \subseteq \mathfrak{A}_{\phi}$.

Proposition 6. If $\phi$ is an element of $X$ of type $2 \mathrm{~b}$ or of type 4b then $\Re \subseteq \mathfrak{U}_{\phi}$.

Proof. From the remarks above, it is sufficient to show that $\phi$ arises from an atom. In the case that $\phi$ is type $2 \mathrm{~b}$, let $P=E_{+}-E_{-}$. Any projection in ker $\phi$ is a sub-projection of $E_{-}$and hence orthogonal 
to $P$; any projection in coker $\phi$ contains $E_{+}$and hence $P$; thus $P$ is an atom. It is evident that $\phi(E)=1$ if and only if $P \leqq E$, and so $\mathfrak{R} \subseteq \mathfrak{A}_{\phi}$.

Now suppose $\phi$ is type $4 \mathrm{~b}$. Let $P=E_{+}-\left(E_{+}\right) \wedge\left(E_{-}\right)$. Since $P$ is orthogonal to $E_{-}$, it follows again that $P$ is orthogonal to each projection in $\operatorname{ker} \phi$, while $P \leqq E_{+}$again implies $P$ is contained in cach projection in coker $\phi$. Here, too, $\phi$ arises from the atom $P$ and $\Re \cong \mathfrak{A}_{\phi}$.

COROLLORY 7. If $\mathfrak{Q}$ is a finite lattice then $\mathfrak{A}=\mathfrak{A} l \mathfrak{l g}(\mathfrak{Q})$ satisfies the radical condition.

Proof. Since $\bigcap_{\phi \in X} \mathfrak{A}_{\phi} \subseteq \mathfrak{R}$, we need only show $\mathfrak{R} \subseteq \mathfrak{A}_{\phi}$, for each $\phi \in X$. Since the lattice is finite, $\phi\left(E_{+}\right)=\Lambda\{\phi(F) \mid \phi(F)=1\}=1$ and $\phi\left(E_{-}\right)=\mathrm{V}\{\phi(E) \mid \phi(E)=0\}=0$. Hence $\phi$ is either type $2 \mathrm{~b}$ or $4 \mathrm{~b}$ and, in either case, $\Re \subseteq \mathfrak{A}_{\phi}$.

In the case in which $\mathfrak{R}$ is a finite lattice, $\Re$ is the ideal of all operators $T$ in $\mathfrak{A}$ such that $P T P=0$ for each of the finitely many atoms for 2 .

REMARK. We conclude this section with a few general comments which will prove useful later. If $\mathbb{R}$ is a commutative subspace lattice acting on $\mathfrak{S}$ and $\mathfrak{U}=\mathfrak{X} l \mathfrak{g} \mathfrak{L}$, let $\mathfrak{E} \mathbb{R}=\{E \mid I-E \in \mathfrak{R}\}$. Then $\mathfrak{E} \mathbb{R}$ is again a commutative subspace lattice and $\mathscr{A Y S}(\mathbb{E} \mathbb{R})=\mathfrak{A}^{*}$. If $\phi$ is a lattice homomorphism of $\&$ onto 2 , define $\phi^{*}$ : $\mathfrak{E} \rightarrow 2$ by $\phi^{*}(E)=1-\phi(1-E)$, for all $E \in \mathbb{E} \Omega$. The mapping $\phi \rightarrow \phi^{*}$ is a bijection of $X(\&)$ onto $X(\mathbb{E} \&)$. It is not hard to show that the radical of $\mathfrak{U}^{*}$ is $\mathfrak{R}^{*}$, (where $\mathfrak{R}$ is the radical of $\mathfrak{X})$, and that $\left(\mathfrak{U}^{*}\right)_{\phi^{*}}=\left(\mathfrak{A}_{\phi}\right)^{*}$. As a consequence, $\mathfrak{R} \cong \mathfrak{A}_{\phi}$

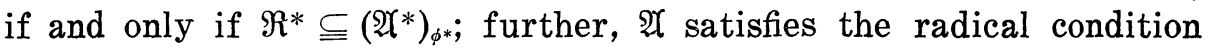
if and only if $\mathfrak{U}^{*}$ does. It is also easy to see that if $k$ is any of $1,2,3,4, \mathrm{~b}$, c, then $\phi$ is type $k$ if and only if $\phi^{*}$ is. Finally, $\phi$ is type a (resp. d) if and only if $\phi^{*}$ is type d (resp. a).

3. Algebras which satisfy the radical condition. We begin this section with a technical lemma. This lemma contains a simplified and generalized version of the essential ingredients in Ringrose's proof that any nest algebra satisfies the radical condition.

Lemma 8. Let $T$ be an operator in $\mathfrak{X}$ and let $\left(P_{n}\right), n=1,2, \cdots$, be a sequence of mutually orthogonal interval projections in $\mathfrak{A}$ such that the following conditions are satisfied.

(i) If $m<n$, then $P_{m} Q P_{n}=0$, for all $Q \in \mathfrak{A}$.

(ii) For any $R \in \mathfrak{B}(\mathfrak{Y}), R=P_{n+1} R P_{n}$ implies $R \in \mathfrak{A}$.

(iii) There exists a number $\lambda>0$ such that $\left\|P_{n} T P_{n}\right\|>\lambda$, for all $n$. 
Then there exists an element $S$ in $\mathfrak{A}$ such that $S T$ is not quasinilpotent. In particular, $T$ does not lie in the radical $\Re$ of $\Re$.

Proof. If the conclusion is true for a nonzero scalar multiple of $T$ then it is also true for $T$. Hence, replacing $T$ by a scalar multiple if necessary, we may assume $\lambda=1$.

For convenience, we henceforth use the same symbol to denote both an orthogonal projection and its range.

For each $n>0$, there exists a unit vector, $x_{n} \in P_{n}$, such that $\left\|P_{n} T x_{n}\right\|>1$. Let $y_{n}=P_{n} T x_{n}$ and let $S_{n}=\left\|y_{n}\right\|^{-2} y_{n} \otimes x_{n+1}$. (The operator $y_{n} \otimes x_{n+1}$ is defined by $\left(y_{n} \otimes x_{n+1}\right)(x)=\left(x, y_{n}\right) x_{n+1}$, for all $x \in \mathfrak{F}_{\text {.) }}$ Observe that $\left\|S_{n}\right\|=\left\|y_{n}\right\|^{-2}\left\|y_{n}\right\|\left\|x_{n+1}\right\|=\left\|y_{n}\right\|^{-1}<1$, and that, since $S_{n}=P_{n+1} S_{n} P_{n}$, each $S_{n} \in \mathfrak{X}$. From the fact that the $P_{n}$ are mutually orthogonal, it follows that the sum $\sum_{n=1}^{\infty} S_{n}$ converges in the strong operator topology to an operator $S$ which lies in $\mathfrak{A}$ and has norm equal to $\sup \left\{\left\|S_{n}\right\|\right\} \leqq 1$.

Let $Q_{n}=\sum_{k \geq n} P_{k}$, for each $n=1,2, \cdots$. Then, if $n>m$, we have $Q_{n} Q_{m}=Q_{n}$. We also have $S=S Q_{1}$ and $S Q_{n}=Q_{n+1} S Q_{n}=Q_{n+1} S$, for all $n$. Since $P_{j} T P_{n}=0$ whenever $j<n$, it follows that $Q_{1} T P_{n}=$ $Q_{n} T P_{n}$, and hence $Q_{1} T Q_{n}=Q_{n} T Q_{n}$, for each $n$.

We claim that, for each $n$ there exists a vector $z_{n+1} \in Q_{n+2}$ such that $(S T)^{n} x_{1}=x_{n+1}+z_{n+1}$. Verification of this claim will prove the lemma; indeed, since $z_{n+1}$ is orthogonal to $x_{n+1}$, we have $\left\|(S T)^{n} x_{1}\right\| \geqq$ $\left\|x_{n+1}\right\| \geqq 1$, whence $\left\|(S T)^{n}\right\| \geqq 1$. Thus $S T$ is not quasi-nilpotent. The claim is proven by an induction argument. First observe that $T x_{1}=y_{1}+a_{1}$, where $a_{1} \perp P_{1}$ (since $y_{1}=P T x_{1}$ ). Then $S y_{1}=x_{2}$ and $S a_{1}=S Q_{1} a_{1}=S Q_{2} a_{1}=Q_{3} S Q_{2} a_{1}$. So if $z_{2}=S a_{1} \in Q_{3}$, then $S T x_{1}=x_{2}+z_{2}$. Next assume $(S T)^{n-1} x_{1}=x_{n}+z_{n}$, with $z_{n} \in Q_{n+1}$. Then $T x_{n}=y_{n}+a_{n}$, where $a_{n} \perp P_{n}$. Since $Q_{1} T P_{n}=Q_{n} T P_{n}$, we obtain $Q_{1} a_{n}=Q_{n+1} a_{n}$. Hence $S a_{n}=S Q_{1} a_{n}=S Q_{n+1} a_{n}=Q_{n+2} S a_{n}$. Also, $S T z_{n}=S Q_{1} T Q_{n+1} z_{n}=$ $S Q_{n+1} T Q_{n+1} z_{n}=Q_{n+2} S T z_{n}$. Therefore,

$$
\begin{aligned}
(S T)^{n} x_{1} & =S T x_{n}+S T z_{n}=S y_{n}+S a_{n}+S T z_{n} \\
& =x_{n+1}+Q_{n+2}\left(S a_{n}+S T z_{n}\right) .
\end{aligned}
$$

Take $z_{n+1}=S a_{n}+S T z_{n}=Q_{n+2}\left(S a_{n}+S T z_{n}\right)$ to complete the proof of the lemma.

With aid of Lemma 8, we sketch a short proof of Ringrose's theorem (cf. [4]).

THEOREM 9 (Ringrose). Every nest algebra satisfies the radical condition.

Proof. If $\&$ is a nest and $\phi \in X(\Omega)$, then $\phi=\phi_{E}^{+}$or $\phi=\phi_{E}^{-}$, for 
some $E \in \mathbb{R}$. The two possibilities are interchanged in the natural correspondence between $X(\mathbb{R})$ and $X(\mathbb{S} \&)$, so it suffices to consider $\phi=\dot{\phi}_{\bar{E}}$ only. If $E$ has an immediate predecessor, then $\phi$ is type $2 \mathrm{~b}$, and $R \subseteq \mathfrak{A}_{\phi}$. Assume $E$ has no immediate predecessor and $T \notin \mathfrak{U}_{\dot{\phi}}$. Then there exists a number, $\lambda>0$, such that $\|(E-F) T(E-F)\|>\lambda$, for all $F<E$. Further, $E$ is a strong limit of projections $F<E$. Hence, if $F_{1}<E$, there exists $F_{2}$ such that $F_{1}<F_{2}<E$ and $\left\|\left(F_{2}-F_{1}\right) T\left(F_{2}-F_{1}\right)\right\|>\lambda$. Indeed, continuing inductively, we can find a sequence $F_{1}<F_{2}<F_{3}<\cdots<E$ of projections such that the intervals $P_{i}=F_{i+1}-F_{i}$ satisfy the hypotheses of Lemma 8. Consequently, $T \notin \Re$ and we have $\mathfrak{R} \subseteq \mathfrak{A}_{\phi}$. This proves the theorem.

Suppose $\mathbb{R}_{1}, \mathfrak{R}_{2}, \mathfrak{R}_{3}, \cdots$ is a finite or countable sequence of commutative subspace lattices. Define the product lattice $\&$ to be the Cartesian product of the $\mathfrak{\&}_{i}$ with co-ordinatewise lattice operations. We may realize $\&$ as a commutative subspace lattice as follows: let $\mathfrak{S}_{i}$ be the Hilbert space on which each $\mathfrak{L}_{i}$ acts. Let $Q_{i}$ be orthogonal projection of $\mathfrak{S}_{\mathfrak{Z}}=\sum_{i}^{\oplus \mathfrak{S}_{i}}$ onto $\mathfrak{S}_{i}$. Then $\{E \mid E$ is a projection in $\mathfrak{B}(\mathfrak{S})$ and each $Q_{i} E \mid \mathscr{S}_{i}$ lies in $\mathfrak{R}_{i}$ \} is a commutative subspace lattice which is lattice isomorphic to 2 . We take this lattice as the subspace lattice direct sum of the $\mathfrak{L}_{i}$. If, on the other hand, $\mathfrak{L}^{\prime}$ is any commutative subspace lattice which is lattice isomorphic to $\mathbb{R}$, let $Q_{i}$ denote the element of $\Omega^{\prime}$ which corresponds under the isomorphism to the element of $\mathbb{R}$ which is $I$ in the $i$ th co-ordinate and 0 in all other co-ordinates. Then the compression of $\&$ to $Q_{i} \mathscr{H}_{\mathcal{E}}$ is a commutative subspace lattice $\mathfrak{R}_{i}^{\prime}$ which is lattice isomorphic to $\mathfrak{R}_{i}$, and $\mathbb{R}^{\prime}$ is the subspace lattice direct sum of the $\mathbb{R}_{\imath}^{\prime}$.

If $\Omega_{1}, \Omega_{2}, \cdots$ are commutative subspace lattices, if $\mathbb{Q}$ is the subspace lattice direct sum of the $\mathfrak{R}_{i}$, if $\mathfrak{A}=\mathfrak{A} \lg \mathfrak{\&}$ and $\mathfrak{A}_{i}=\mathfrak{A l g} \mathfrak{S}_{i}$, for each $i$, then it is clear that $\mathfrak{A}=\sum_{i}^{\oplus \mathfrak{A}}{ }_{i}$.

Theorem 10 below proves that if $\mathfrak{A}$ is a finite direct sum of algebras, each of which satisfies the radical condition, then $\mathfrak{U}$ also satisfies the radical condition. Actually, a bit more is true. Let us say that a commutative subspace lattice $\mathbb{Q}$ satisfies the radical condition concretely provided that $\mathfrak{U} I g \&$ satisfies the radical condition and that $\&$ satisfies the radical condition universally provided that भIfg $\mathfrak{L}^{\prime}$ satisfies the radical condition whenever $\mathfrak{L}^{\prime}$ is a commutative subspace lattice which is lattice isomorphic to $\mathbb{L}$. Theorem 10 essentially proves that if $\Omega_{1}, \cdots, \Omega_{n}$ satisfy the radical condition universally, then the product lattice $\mathbb{Z}$ also satisfies the radical condition universally. We remark in passing that the following natural question remains open: if $\mathfrak{L}$ satisfies the radical condition concretely, does it satisfy the radical condition universally? The question arises from that fact that if $\mathbb{Z}$ and $\mathfrak{L}^{\prime}$ are lattice isomorphic but not unitarily equivalent, then $\mathfrak{A l g} \mathfrak{L}$ and $\mathfrak{U} \lg \mathfrak{L}^{\prime}$ need not be algebraically 
isomorphic.

THeOREM 10. Let $\mathfrak{\Omega}_{1}, \cdots, \mathfrak{\Omega}_{n}$ be commutative subspace lattices such that each $\mathfrak{A l g}\left(\mathbb{R}_{i}\right), i=1, \cdots, n$, satisfies the radical condition. Let $\&$ be the subspace lattice direct sum of the $\mathbb{R}_{i}$. Then $\mathfrak{A I g}^{2}$ satisfies the radical condition.

Proof. Let $\mathscr{S}_{i}$ be the Hilbert space on which $\mathfrak{S}_{i}$ acts, for $i=$ $1, \cdots, n$. Then $\&$ acts on $\mathfrak{F}_{\mathcal{E}}=\sum_{i}^{\oplus} \mathfrak{S}_{i}$ and $\&$ contains each $Q_{i}$, where $Q_{i}$ is orthogonal projection of $\mathscr{S}$ onto $\mathfrak{S}_{i}$. Let $\phi \in X(\mathbb{R})$ and suppose $T \notin \mathfrak{A}_{\phi}$. Since the $Q_{i}$ are mutually orthogonal intervals from $\mathbb{\&}$ whose sum is $I$, one of the $Q_{i}$ 's is a test interval for $\phi$. Let $i$ be the unique index such that $\phi\left(Q_{i}\right)=1$. Define a lattice homomorphism $\phi_{0} \in X\left(\Omega_{i}\right)$ by $\phi_{0}(E)=\phi(E)$. (Each projection $E$ in $\Omega_{i}$ may be extended to a projection in $\mathbb{L}$ by defining it to be 0 on $\sum_{j \neq i}^{\oplus} \mathfrak{S}_{j}$.) Let $T_{0}=$ $Q_{i} T Q_{i}$. It then follows that $T_{0} \notin\left(\mathfrak{A}_{i}\right)_{\phi_{0}}$. Consequently, there is an operator, $S \in \mathfrak{A}_{i}$, such that $S T_{0}$ is not quasi-nilpotent. We may extend $S$ to an operator in $\mathfrak{A}$ be defining it to be 0 on $\sum_{j \neq 2}^{\oplus} \mathfrak{F}_{j}$; then $S Q_{i} T Q_{i}$ is not quasi-nilpotent in $\mathscr{A}$ and so $Q_{i} T Q_{i}$ does not lie in the radical, $\Re$, of $\mathfrak{A}$. Since $\Re$ is a two-sided ideal, $T \notin \Re$. Thus $\Re \subseteq \mathfrak{A}_{\phi}$, for all $\phi \in X(\Re)$ and $\mathscr{U} l g \mathfrak{g}$ satisfies the radical condition.

THEOREM 11. Let $\mathfrak{\Omega}_{1}, \mathfrak{\Omega}_{2}, \cdots$, be a sequence of totally ordered commutative subspace lattices. Let $\&$ be the subspace lattice direct sum of the $\mathfrak{\Omega}_{2}$. Then $\mathfrak{2}$ Ilg \& satisfies the radical condition.

Proof. Let $\mathscr{S}_{2}$ denote the Hilbert space on which each $\mathfrak{R}_{i}$ acts. $\mathfrak{Z}$ acts on $\mathfrak{S}=\sum_{i}^{\oplus} \mathfrak{A}_{i}$. Each $\mathfrak{A}_{\imath}=\mathfrak{A l l}_{\mathfrak{l}}\left(\mathfrak{R}_{i}\right)$ is a nest algebra and $\mathfrak{A}=$ $\mathfrak{U}$ Ilg $\mathbb{L}=\sum_{i}^{\oplus} \mathfrak{A}_{i}$. Let $Q_{i}$ denote orthogonal projection of $\mathfrak{S}_{\mathcal{C}}$ onto $\mathfrak{F}_{i}$. Note that, for any nonempty subset $K \subseteq N, \sum_{j \in K} Q_{j}$ is a projection in 2. Denote this projection by $Q(K)$. Each $Q(K)$ is an interval from \&. Fix a lattice homomorphism $\phi \in X(\&)$. If one of the $Q_{i}$ is a test interval for $\phi$, then we may argue exactly as in Theorem 10 to show $\Re \subseteq \mathfrak{A}_{\phi}$. ( $\Re$ is the radical of $\mathscr{A}$.) Since there are infinitely many $Q_{i}$, it may be that none of them is a test interval for $\phi$.

Let $\widetilde{\mho}$ be the family of all nonempty subsets, $K$, of $N$, such that $Q(K)$ is a test interval for $\phi$. If $K_{1}$ and $K_{2}$ lie in $\mathfrak{F}$, then $\dot{\phi}\left(Q\left(K_{1} \cap K_{2}\right)\right)=\phi\left(Q\left(K_{1}\right) \wedge Q\left(K_{2}\right)\right)=1 \wedge 1=1$, hence $K_{1} \cap K_{2} \in \widetilde{F}$. Also, if $K_{1} \in \mathfrak{F}$ and $K_{2} \supseteqq K_{1}$, then $Q\left(K_{2}\right) \geqq Q\left(K_{1}\right)$, hence $\phi\left(Q\left(K_{2}\right)\right)=1$. So $K_{2} \in \widetilde{F}$ also, and we see that $\mathfrak{F}$ is a filter. Finally, if $K_{1} \cup K_{2} \in \mathfrak{F}$ then $\phi\left(Q\left(K_{1}\right) \vee Q\left(K_{2}\right)\right)=\phi\left(Q\left(K_{1} \cup K_{2}\right)\right)=1$, whence one of $K_{1}$ and $K_{2}$ must $b$ : in $\widetilde{\Re}$. Thus $\widetilde{F}$ is an ultrafilter. (It is not difficult, by the way, to cinstruct at least one lattice homomorphism $\phi$ for each ultrafilter $\widetilde{F}$ on $N$.) 
If one of the $Q_{i}$ is a test interval for $\phi$, then $\mathfrak{F}$ is the family of all subsets of $N$ which contain $i$. Assume henceforth that no $Q_{i}$ is a test interval for $\phi$; consequently each set in $\widetilde{f}$ is infinite. Now suppose $T \notin \mathfrak{A}_{\phi}$. Since $N_{\phi}(T)>0$, we may, without loss of generality, assume $N_{\phi}(T)>1$. Let $\lambda$ be a number such that $N_{\dot{\phi}}(T)>\lambda>1$. Let $K=\left\{i \mid\left\|Q_{i} T Q_{i}\right\|>\lambda\right\}$. Using the fact that each $Q_{i}$ is reducing for $T$, we have $\|Q(N-K) T Q(N-K)\|=\sup _{i \notin K}\left\|Q_{i} T Q_{i}\right\| \leqq \lambda$. Since $N_{\phi}(T)>\lambda$, it follows that $Q(N-K)$ is not a test interval for $\phi$, and hence that $Q(K)$ is. Thus $K \in \widetilde{F}$ and, in particular, $K$ is infinite. Suppose there is no upper bound for the set of integers, $n$, for which there exist some index $i \in K$ and some operator $S_{i} \in \mathfrak{A}_{i}$, with $\left\|S_{i}\right\| \leqq 1$, such that $\left\|T_{i}\left(S_{i} T_{i}\right)^{n}\right\| \geqq 1$. (Here, $T_{i}$ denotes the restriction to $\mathfrak{S}_{i}$ of $Q_{i} T$. Clearly, $T_{i} \in \mathfrak{A}_{i}$.) Then for each $i \in K$, choose a positive integer $n_{i}$ and an operator $S_{i} \in \mathfrak{N}_{i}$, with $\left\|S_{i}\right\| \leqq 1$, so that:

(i) $\sup \left\{n_{i} \mid i \in K\right\}=\infty$

(ii) $\left\|T_{i}\left(S_{i} T_{i}\right)^{n_{i}}\right\| \geqq 1$, for all $i \in K$.

(If conditions (i) and (ii) cannot be obtained, it means that there exists a finite subset, $K_{0}$, of $K$ such that, if $K_{0}$ is deleted from $K$, then there is an upper bound on the set of integers, $n$, with the required properties. Whenever this is the case, the proof preceeds as in the following paragraph, with $K$ replace by $K-K_{0}$. Since $K_{0}$ is finite, $Q\left(K-K_{0}\right)$ is a test interval for $\left.\phi.\right)$ If $i \notin K$, let $S_{\imath}=0$. Let $S=\sum_{\imath}^{\oplus} S_{i}$. Then $S$ is bounded $\left(\|S\|=\sup _{i}\left\|S_{i}\right\| \leqq 1\right)$ and, for each $i \in K,\left\|T(S T)^{n_{i}}\right\| \geqq\left\|T_{i}\left(S_{i} T_{i}\right)^{n_{i}}\right\| \geqq 1$. Hence $1 \leqq\|T\|\left\|(S T)^{n_{2}}\right\|$, for each $n_{i}$. Therefore $\left\|(S T)^{n_{i}}\right\|^{1 / n_{i}} \geqq(1 /\|T\|)^{1 / n_{i}}$, for each $n_{i}$. Since the $n_{i}$ are unbounded, we obtain $\lim _{n \rightarrow \infty}\left\|(S T)^{n}\right\|^{1 / n} \geqq 1$. Hence $S T$ is not quasi-nilpotent and thus $T \notin \Re$.

We shall now assume that $T \in \mathfrak{R}$ and show that this leads to a contradiction. From what has just been proven, we know that there exists an integer, $n$, such that $\left\|T_{i}\left(S_{i} T_{i}\right)^{n}\right\|<1$, for all $i \in K$, and for all $S_{i} \in \mathfrak{N}_{i}$, with $\left\|S_{i}\right\| \leqq 1$. We need the following lemma:

LEMMA 12. Let $\mathfrak{\Omega}_{0}$ be a complete nest and let $\mathfrak{A}_{0}=\mathfrak{U l l}_{\mathrm{Ug}} \mathfrak{\Omega}_{0}$ be the associated nest algebra. Assume $R \in \mathfrak{A}_{0},\|R\|=\mu>1$, and $R$ belongs to the radical of $\mathfrak{A}_{0}$. Assume further that $\left\|R(S R)^{n}\right\|<1$, for all $S \in \mathfrak{A}_{0}$ with $\|S\| \leqq 1$. Then there exist $n+1$ disjoint intervals, $P_{k}$, from $\mathfrak{S}_{0}$ such that $I=\sum_{k=1}^{n+1} P_{k}$ and $\left\|P_{k} R P_{k}\right\| \leqq 1$, for each $k=1, \cdots, n+1$.

Proof. We shall assume that the required intervals do not exist and deduce a contradiction. As a first step, observe that there exist projections $I=E_{1}>E_{2}>\cdots>E_{n+2}>0$ in \& such that

(i ) $\left\|\left(E_{k}-E_{k+1}\right) R\left(E_{k}-E_{k+1}\right)\right\| \leqq 1$, for $k=1, \cdots, n+1$.

(ii) If $F<E_{k+1}$ then $\left\|\left(E_{k}-F\right) R\left(E_{k}-F\right)\right\|>1$, for $k=1, \cdots$, $n+1$. 
Indeed, set $E_{1}=I$ and assume inductively that $E_{1}, \cdots, E_{k}>0$ have been constructed satisfying these conditions. Let

$$
E_{k+1}=\inf \left\{F \in \mathfrak{Q} \mid F<E_{k} \text { and }\left\|\left(E_{k}-F\right) R\left(E_{k}-F\right)\right\| \leqq 1\right\} \text {. }
$$

The set over which the infimum is taken is nonempty (since $R$ belongs to the radical of $\mathfrak{A}$ ), is strongly closed, and contains $E_{k+1}$ as a limit point. This shows that $E_{k+1}$ satisfies condition (i), while condition (ii) follows automatically from the definition of $E_{k+1}$. Further, as long as $k \leqq n+1$, we must have $E_{k+1} \neq 0$, for otherwise the projections $P_{j}=E_{j}-E_{j+1}, j=1, \cdots, k$, would satisfy the conclusion of the lemma. Thus we may construct inductively the desired $n+2$ projections in $\mathbb{Z}$ satisfying (i) and (ii).

Observe that, since $R$ lies in the radical of $\mathfrak{A}$, if $P$ is any atom from $\mathfrak{R}$, then $P R P=0$. Consequently, if $E \in \mathbb{R}$ and if $F$ is an immediate predecessor of $E$, then $R E=F R E$.

Next construct projections $F_{1}>F_{2}>\cdots>F_{n+1}$ and vectors $x_{1}, x_{2}, \cdots, x_{n+1}$ satisfying:

(iii) $F_{k}<E_{k}$ for all $k=1, \cdots, n+1$, and, if $E_{k}$ has an immediate predecessor, then $F_{k}$ is the immediate predecessor of $E_{k}$.

(iv) $\left\|x_{k}\right\|=1$, for all $k$.

(v) Each $x_{k} \in E_{k}-F_{k+1}$. Further, if $E_{k}$ has no immediate predecessor, then $x_{k} \in F_{k}-F_{k+1}$; if $E_{k+1}$ has an immediate predecessor, then $x_{k} \in E_{k}-E_{k+1}$.

(vi) $\left\|R x_{n+1}\right\|>1$ and $\left\|\left(E_{k}-F_{k+1}\right) R x_{k}\right\|>1$, for all $k=1, \cdots, n$.

Indeed, from the construction of $E_{1}, \cdots, E_{n+2}$ we know that $\left\|E_{n+1} R E_{n+1}\right\|>1$. If $E_{n+1}$ has an immediate predecessor, let $F_{n+1}$ be that immediate predecessor and let $x_{n+1}$ be any unit vector in $E_{n+1}$ such that $\left\|E_{n+1} R x_{n+1}\right\|>1$. If $E_{n+1}$ has no immediate predecessor, then $E_{n+1}$ is a strong limit of projections in $\&$ which are strictly less than $E_{n+1}$. Hence there exists a projection $F_{n+1}<E_{n+1}$ in $\mathbb{L}$ such that $\left\|F_{n+1} R F_{n+1}\right\|>1$. Let $x_{n+1}$ be a unit vector in $F_{n+1}$ such that $\left\|F_{n+1} R x_{n+1}\right\|>1$.

Suppose projections $F_{k+1}, \cdots, F_{n+1}$ and vectors $x_{k+1}, \cdots, x_{n+1}$ have been constructed satisfying (iii)-(vi). We construct $F_{k}$ and $x_{k}$ as follows; since $F_{k+1}<E_{k+1},\left\|\left(E_{k}-F_{k+1}\right) R\left(E_{k}-F_{k+1}\right)\right\|>1$. If $E_{k}$ has an immediate predecessor, we set $F_{k}$ equal to the immediate predecessor and let $x_{k}$ be any unit vector in $E_{k}-F_{k+1}$ such that $\left\|\left(E_{k}-F_{k+1}\right) R x_{k}\right\|>$ 1. If $E_{k}$ has no immediate predecessor, then there exists a projection $F_{k}<E_{k}$ in $\mathbb{R}$ such that $\left\|\left(F_{k}-F_{k+1}\right) R\left(F_{k}-F_{k+1}\right)\right\|>1$. Let $x_{k}$ be a unit vector in $F_{k}-F_{k+1}$ such that $\left\|\left(F_{k}-F_{k+1}\right) R x_{k}\right\|>1$. In the event $F_{k+1}$ is the immediate predecessor of $E_{k+1}$, we have $\left(E_{k+1}-F_{k+1}\right) R\left(E_{k+1}-F_{k+1}\right)=0$. Consequently, 


$$
\left(E_{l}-F_{k+1}\right) R x_{k}=\left(E_{k}-F_{k+1}\right) R\left(E_{k}-E_{k+1}\right) x_{k} .
$$

Therefore, we may assume, without loss of generality, that $x_{k} \in E_{k}$ $E_{k+1}$, whenever $E_{k+1}$ has an immediate predecessor.

Note that, regardless of whether or not $E_{k}$ has an immediate predecessor, $R x_{k} \in F_{k}, k=1, \cdots, n+1$. For each $k=1, \cdots, n$, let $y_{k}=\left(F_{k}-F_{k+1}\right) R x_{k}$. By (vi), $\left\|y_{k}\right\|>1$, for all $k$. Hence the operator $S_{k}=\left\|y_{k}\right\|^{-2} y_{k} \otimes x_{k+1}$ has norm less than one. Let $S=\sum_{k=1}^{n} S_{k}$. It is clear that the $\left\{y_{k}\right\}$ form an orthogonal set of vectors. To see that the $\left\{x_{k}\right\}$ also form an orthogonal set of vectors, it is in this case sufficient to show that $x_{k} \perp x_{k+1}$, for each $k=1, \cdots, n+1$. If $E_{k+1}$ has an immediate predecessor then $x_{k} \in E_{k}-E_{k+1}$ and $x_{k+1} \in E_{k+1}$, hence they are orthogonal. If $E_{k+1}$ has no immediate predecessor, then $x_{k} \in E_{k}-F_{k+1}$ and $x_{k+1} \in F_{k+1}$, and again they are orthogonal. As a consequence of the fact that the families $\left\{y_{k}\right\}$ and $\left\{x_{k}\right\}$ are orthogonal, we obtain $\|S\|=\sup _{k}\left\{\left\|S_{k}\right\|\right\}<1$.

Finally, we claim that $(S R)^{n} x_{1}=x_{n+1}$. This will prove the lemma, since $\left\|R(S R)^{n} x_{1}\right\|=\left\|R x_{n+1}\right\|>1$ implies $\left\|R(S R)^{n}\right\|>1$, a contradiction. To prove the claim argue much as in Lemma 8: show inductively that for $2 \leqq k \leqq n,(S R)^{k-1} x_{1}=x_{k}+b_{k}$, where $b_{k} \in E_{k+1}$ if $E_{k+1}$ has an immediate predecessor and $b_{k} \in F_{k+1}$ otherwise (and in either case, $\left.R b_{k} \in F_{k+1}\right)$, and also that $R(S R)^{k-1} x_{1}=y_{k}+a_{k}$, where $a_{k} \in F_{k+1}$. In the final step, $S a_{n}=0$, since $S F_{n+1}=0$; hence $(S R)^{n} x_{1}=x_{n+1}$, as required.

We now return to the proof of theorem. Recall that $T$ is an operator in $\Re$ which is not in $\mathfrak{U}_{\phi}$ and that $n$ is an integer such that, for all $i \in K,\left\|T_{i}\left(S_{i} T_{i}\right)^{n}\right\|<1$, for all $S_{i} \in \mathfrak{A}_{i}$ with $\left\|S_{i}\right\| \leqq 1$. From Lemma 12, it follows that for each $i \in K$, there exist $n+1$ disjoint intervals $P_{i}^{(k)}$, from $\mathbb{S}_{i}$, such that $Q_{i}=\sum_{k=1}^{n+1} P_{i}^{(k)}$ and $\left\|P_{i}^{(k)} T_{i} P_{i}^{(k)}\right\| \leqq 1$, for each $k=1, \cdots, n+1$. Let $P^{(k)}=\sum_{i \in K}^{\oplus} P_{i}^{(k)}$. Each $P^{(k)}$ is an interval from 2 , the $P^{(k)}$ are mutually orthogonal, and $Q(K)=$ $\sum_{k=1}^{n+1} P^{(k)}$. Since $Q(K)$ is a test interval for $\phi$, one of the $P^{(k)}$ is also a test interval for $\phi$. But $\left\|P^{(k)} T P^{(k)}\right\|=\sup _{i \in K}\left\|P_{i}^{(k)} T_{i} P_{i}^{(k)}\right\| \leqq 1$, for each $k$. Hence $N_{\phi}(T) \leqq 1$, a contradiction. Thus, $T \notin \mathfrak{A}_{\phi}$ implies $T \notin \Re$ and we obtain $\Re \subseteq \mathfrak{U}_{\phi}$. This proves the theorem.

We have yet to provide examples of lattice homomorphisms of types $3 \mathrm{a}, 3 \mathrm{c}, 3 \mathrm{~d}$, and $4 \mathrm{~b}$. The class of examples described next yields homomorphisms of types $3 \mathrm{a}$ and $4 \mathrm{~b}$. The adjoint algebras yield homomorphisms of type $3 \mathrm{~d}$, while type $3 \mathrm{c}$ may be obtained by means of a variant construction. The lattice in the examples which follow may be considered to be the tensor product of the lattice $N \cup\{\infty\}$ with itself.

Let $Y=N \times N$ be provided with the product order: $(n, m) \leqq$ $(p, q)$ if and only if $n \leqq p$ and $m \leqq q$. Define a subset $S \leqq Y$ to 
be increasing if $x \in S$ and $y \geqq x$ imply $y \in S$. For each $x \in Y$, let $\mathfrak{S}_{x}$ be a separable Hilbert space and let $\mathfrak{S}=\sum_{x \in Y}^{\oplus} \mathfrak{F}_{x}$. Identify each subset $S \subseteq Y$ with the subspace $\sum_{x \in S}^{\oplus} \mathfrak{F}_{x}$ of $\mathscr{S}$ and with the orthogonal projection of $\mathfrak{S}$ onto that subspace. A projection associated with a subset $S$ is said to be increasing whenever $S$ is an increasing set. The family $\&$ of increasing projections is a commutative subspace lattice. Let $\mathfrak{A}=\mathfrak{A l g} \mathfrak{R}$.

Proposition 13. $\mathfrak{A}$ satisfies the radical condition.

Proof. In order to prove the proposition we must show that $\Re \subseteq \mathfrak{A}_{\phi}$, for any lattice homomorphism $\phi$ in $X$. We already know this to be true if $\phi$ is of type $2 \mathrm{~b}$ or $4 \mathrm{~b}$. (Actually, there are no lattice homomorphisms of $\&$ of type $2 \mathrm{~b}$ - but we do not need this fact.) Of the other possible types, only types la and 3a can occur, and these can be handled with the aid of Lemma 8 . The following notation will be helpful: let

$$
\begin{aligned}
& H_{n}=\{(p, q) \in Y \mid q \geqq n\} \\
& V_{n}=\{(p, q) \in Y \mid p \geqq n\} \\
& E_{x}=\{y \in Y \mid y \geqq x\} .
\end{aligned}
$$

Each of these symbols denotes both an increasing set and a projection in $\mathbb{2}$.

Lemma 14. Let $\phi \in X$. Then $\phi\left(E_{-}\right)=0$ and, further, if $\phi$ is not of type $4 \mathrm{~b}$, then $E_{-}$is either 0 or $H_{n}$ or $V_{n}$, for some $n \geqq 2$.

Proof. There is nothing to prove if $E_{-}=0$, so assume $E_{-}>0$. Hence there is some nonzero projection $F$ in \& such that $\phi(F)=0$. In particular, by choosing a point $x=(m, n)$ in $F$, we have that $\phi\left(E_{x}\right)=0$. Since $H_{n} \wedge V_{m}=E_{x}$, at least one of $H_{m}$ and $V_{m}$ lies in ker $\phi$. If both do, then $G=H_{n} \vee V_{m} \in \operatorname{ker} \phi$. Hence $I-G$ is a test interval for $\phi$; since $I-G$ can be written as a finite sum of atoms, one of these atoms is a test interval for $\phi$. Therefore $\phi$ must be of type b. The atom is just a singleton $\{(p, q)\}$ (and the corresponding projection); it is clear that $E_{+}=E_{(p, q)}$ and $E_{-}=H_{q+1} \vee V_{p+1}$. (This shows $\phi$ is of type 4). Thus $\phi\left(E_{-}\right)=0$.

Assume henceforth that $\phi$ is not of type $4 \mathrm{~b}$. Then exactly one of $H_{n}$ and $V_{m}$ lies in $\operatorname{ker} \phi$. To fix the argument, let us say that $\phi\left(V_{m}\right)=0$. The considerations above imply that $\phi\left(H_{q}\right)=1$, for all $q$. Therefore $E_{+}=0$ and types $1 \mathrm{~d}$ and $3 \mathrm{~d}$ cannot occur. Now let $y=(p, q)$ be any point of $E_{-}$. Then $y$ must be in some increasing set in the kernel of $\phi$ and, in particular, $\phi\left(E_{y}\right)=0$. Since $\phi\left(H_{q}\right)=1$, 
we have $\phi\left(V_{p}\right)=0$ and $V_{p} \subseteq E_{-}$. Thus $E_{-}$is a union of $V_{p}$ and so $E_{-}=V_{r}$ for some $r$. Since $V_{1}=I$ and $\phi(I)=1, r \geqq 2$. The facts that $\phi\left(H_{s}\right)=1$ and $\phi\left(H_{s} \wedge V_{r}\right)=\phi\left(E_{(s, r)}\right)=0$, for any $s$, imply that $\phi\left(E_{-}\right)=\phi\left(V_{r}\right)=0$. The alternative case, in which $\phi\left(H_{n}\right)=0$ and $\phi\left(V_{m}\right)=1$, yields $E_{-}=H_{r}$ for some $r \geqq 2$ and $\phi\left(E_{-}\right)=0$ in exactly the same fashion. This proves the le nma.

As a consequence of this lemma we have only limited possibilities for $\phi$. If $E_{\sim}=0$, then $\phi(F)=1$ if and only if $F>0$, and $\phi$ is type 1a. This is the only homomorphism of type 1a. If $\phi$ is neither type 4 b nor 1a, then we must have $E_{-}=V_{n}$ or $E_{-}=H_{n}$ for some $n \geqq 2$. In either case, $E_{+}=0$ and $\phi$ is type 3a. Further, we know that $\phi(F)=0$ if and only if $F \leqq E_{-}$. We use Lemma 8 to dispose of the type 1a homomorphism. For each $x=(p, q) \in Y$, let $F_{x}=\{y \in Y \mid y \geqq x\}$. Each $F_{x} \in \mathcal{R}$, and if $p \rightarrow \infty, q \rightarrow \infty$ then $F_{x} \rightarrow 0$ in the strong operator topology. If $x<y$ then $E_{x}-F_{y} \wedge E_{x}$ is an interval from $\&$ corresponding to the "rectangle" $\{z \mid x \leqq z \leqq y\}$. Suppose $T \notin \mathscr{A}_{\phi}$. Then there exists a number $\lambda>0$ such that $N_{\phi}(T)>\lambda$. We construct inductively a sequence $P_{n}$ satisfying the hypotheses of Lemma 8 . Since each $E_{x}$ is a test interval for $\phi,\left\|E_{x} T E_{x}\right\|>\lambda$, for all $x$. Fix $x_{1}=\left(p_{1}, q_{1}\right)$. Since $I-F_{y} \rightarrow I$ strongly as $y \rightarrow(\infty, \infty)$, there exists $y_{1}=\left(r_{1}, s_{1}\right)$ such that, if $P_{1}=E_{x_{1}}-F_{y_{1}} \wedge E_{x_{1}}$, then $\left\|P_{1} T P_{1}\right\|>\lambda$. Suppose $P_{1}, \cdots, P_{n-1}$ are intervals of the form $E_{x}-F_{y} \wedge E_{x}$ which satisfy the hypotheses of Lemma 8. Let $x_{n}=\left(p_{n}, q_{n}\right)$ be such that $p_{n}>r_{n-1}$ and $q_{n}>s_{n-1}$. Choose $y_{n}$ such that $P_{n}=E_{x_{n}}-F_{y_{n}} \wedge E_{x_{n}}$ satisfies $\left\|P_{n} T P_{n}\right\|>\lambda$. In this fashion we obtain a sequence $\left(P_{n}\right)$ of projections satisfying the hypotheses of Lemma 8 ; hence $T \notin \Re$. Thus $\mathfrak{R} \subseteq \mathfrak{A}_{\phi}$.

Finally, assume $\phi$ is type 3 a and $T \notin \mathfrak{A}_{\phi}$. We know that $E_{-}=$ $V_{n}$ or $E_{-}=H_{n}$, for some $n>2$. Let $F=V_{n-1}$ or $F=H_{n-1}$ accordingly. Let $P=F-E_{-}$. Then $\|P T P\| \geqq N_{\phi}(T)>0$. The lattice $P \&$ may be viewed as a commutative subspace lattice on $P F_{2}$; it is clearly a nest. We may identify $P \mathfrak{A} P$ as the nest algebra of this nest. If $\psi$ is the lattice homomorphism $\phi_{0}^{+}$defined on the nest $P 2$, then it follows that $P T \mid P \mathcal{E}$ does not lie in $(P \mathfrak{A} P)_{\psi}$. Since $P \mathfrak{X} P$ is a nest algebra, $P T P$ does not lie in the radical of the algebra. In particular, there is an operator $S \in P \mathfrak{X} P$ such that $S P T P$ is not quasi-nilpotent. Since $S$ may be viewed as an element of $\mathfrak{A}$ also, it follows that $T \notin \Re$. This completes the proof of the proposition.

Remarks. 1. The lattice in the example above is isomorphic to the lattice of all nonincreasing sequences with values in $N \cup\{\infty\}$, where the lattice operations are given by $\left(a_{n}\right) \wedge\left(b_{n}\right)=\left(\max \left(a_{n}, b_{n}\right)\right)$ and $\left(a_{n}\right) \vee\left(b_{n}\right)=\left(\min \left(a_{n}, b_{n}\right)\right)$. 
2. If $\mathfrak{A}$ is a reflexive algebra with subspace lattice $\mathfrak{Z}$ isomorphic to the type above, and if $\phi$ is a lattice homomorphism of type $3 a$, then $\phi^{*}$ is a lattice homomorphism of type $3 \mathrm{~d}$. (cf. the remark at the end of $\S 2$.)

3. We have now displayed an example of a lattice homomorphism of every type excepting type 3c. An example of a homomorphism of type 3c may be obtained by using $Z \times Z$ in place of $N \times N$ in the construction above. Use the product order to define a lattice of "increasing sets" and a commutative subspace lattice on

$$
\Sigma^{\oplus}\left\{\mathfrak{S}_{x} \mid x \in \boldsymbol{Z} \times \boldsymbol{Z}\right\}
$$

in exactly the same way; again let $V_{n}=\{(p, q) \mid p \geqq n\}$ and $H_{n}=$ $\{(p, q) \mid q \geqq n\}$, for each integer $n$. Observe that $\mathbb{B}=\left\{E \in \mathbb{R} \mid E \geqq V_{n}\right.$, for some $n \in \boldsymbol{Z}\}$ is a co-ideal and that $\mathfrak{\Im}=\left\{E \in \mathbb{R} \mid E \leqq H_{n}\right.$, for some $n \in \boldsymbol{Z}\}$ is an ideal. A simple argument from lattice theory guarantees the existence of a prime ideal containing $\mathfrak{\Im}$ whose complement contains (S). Thus there exists an element $\phi \in X$ such that $\phi\left(V_{n}\right)=1$ and $\phi\left(H_{n}\right)=0$, for all $n$. Hence $E_{+} \leqq \Lambda V_{n}=0$ and $E_{-} \geqq \bigvee_{n} H_{n}=I$. Such a $\phi$ is of type 3c.

In the example based on $\boldsymbol{Z} \times \boldsymbol{Z}$ it is precisely homomorphisms of type $3 \mathrm{c}$ which stand in the way of a proof that 2 IIg $\&$ satisfies the radical condition. All other types can be handled in a fashion similar to the homomorphisms in the $N \times N$ example. There are many homomorphisms of type $3 \mathrm{c}$; in fact the cardinality of the set of type $3 \mathrm{c}$ homomorphisms in $2^{\boldsymbol{N}_{0}}$. One large class amenable to analysis has the property that if $T$ is not in $\mathfrak{X}_{\phi}$ for some $\dot{\phi}$ in this class, then the compression of $T$ to some one of the $\mathfrak{S}_{x}$ must be nonzero. Such $T$ cannot lie in the radical (cf. the analysis of type $\mathrm{b}$ homomorphisms). Two more examples (or four if the roles of $H_{n}$ and $V_{n}$ are interchanged) may be obtained by noticing that $\Im$ is a prime ideal and that $\$ 3$ is a prime co-ideal. For either of these, a technical and elaborate extension of lemma 8 can be used to show that $\Re \subseteq \mathfrak{A}_{\dot{\phi}}$. We omit details since they are complicated and do not result in a complete verification of the conjecture. The sticking point is that there exist still other homomorphisms of type $3 \mathrm{c}$ than the ones alluded to above; but we do not have a sufficiently explicit description of them to prove the relation $\Re \subseteq \mathfrak{A}_{\phi}$. A theorem similar to Theorem 11, with the condition "each $\mathfrak{B}_{i}$ is a nest" replaced by "each $\mathfrak{\Omega}_{i}$ is a finite lattice," would be sufficient to permit proof of the conjecture for the $\boldsymbol{Z} \times \boldsymbol{Z}$ example.

\section{REFERENCES}

1. W. Arveson, Operator algebras and invariant subspaces, Ann. of Math., 100 (1974), 
433-532.

2. G. Birkhoff, Lattice Theory, $3^{\text {rd }}$ ed., A.M.S. Colloquium Publications, 25, Providence, R.I.

3. C. Rickart, General Theory of Banach Algebra, Van Nostrand, New York, 1960.

4. J. Ringrose, On some algebras of operators, Proc. London Math. Soc. (3), 15 (1965), 61-83.

Received February 6, 1976. This research was supported in part by the National Research Council of Canada under Grant No. A9227 and by the Research Advisory Board of the University of Guelph under Grant No. GR 23.

UNIVERSITY OF GUELPH

AND

UNiversity of ALABAMa 


\section{PACIFIC JOURNAL OF MATHEMATICS}

\section{EDITORS}

RICHARD ARENS (Managing Editor)

University of California

Los Angeles, California 90024

\section{R. A. Beaumont}

University of Washington

Seattle, Washington 98105
J. DugundjI

Department of Mathematics

University of Southern California

Los Angeles, California 90007

D. Gilbarg and J. Milgram

Stanford University

Stanford, California 94305

\section{ASSOCIATE EDITORS}
E. F. BECKENBACH
B. H. NeumanN
F. WOLF
K. YosHIDA

\section{SUPPORTING INSTITUTIONS}

\author{
UNIVERSITY OF BRITISH COLUMBIA \\ CALIFORNIA INSTITUTE OF TECHNOLOGY \\ UNIVERSITY OF CALIFORNIA \\ MONTANA STATE UNIVERSITY \\ UNIVERSITY OF NEVADA \\ NEW MEXICO STATE UNIVERSITY \\ OREGON STATE UNIVERSITY \\ UNIVERSITY OF OREGON \\ OSAKA UNIVERSITY
}

\author{
UNIVERSITY OF SOUTHERN CALIFORNIA \\ STANFORD UNIVERSITY \\ UNIVERSITY OF HAWAII \\ UNIVERSITY OF TOKYO \\ UNIVERSITY OF UTAH \\ WASHINGTON STATE UNIVERSITY \\ UNIVERSITY OF WASHINGTON \\ AMERICAN MATHEMATICAL SOCIETY
}

The Supporting Institutions listed above contribute to the cost of publication of this Journal, but they are not owners or publishers and have no responsibility for its content or policies.

Mathematical papers intended for publication in the Pacific Journal of Mathematics should be in typed form or offset-reproduced, (not dittoed), double spaced with large margins. Please do not use built up fractions in the text of your manuscript. You may however, use them in the displayed equations. Underline Greek letters in red, German in green, and script in blue. The first paragraph or two must be capable of being used separately as a synopsis of the entire paper. Items of the bibliography should not be cited there unless absolutely necessary, in which case they must be identified by author and Journal, rather than by item number. Manuscripts, in triplicate, may be sent to any one of the editors. Please classify according to the scheme of Math. Reviews, Index to Vol. 39. All other communications should be addressed to the managing editor, or Elaine Barth, University of California, Los Angeles, California, 90024.

The Pacific Journal of Mathematics expects the author's institution to pay page charges, and reserves the right to delay publication for nonpayment of charges in case of financial emergency.

100 reprints are provided free for each article, only if page charges have been substantially paid. Additional copies may be obtained at cost in multiples of 50 .

The Pacific Journal of Mathematics is issued monthly as of January 1966. Regular subscription rate: $\$ 72.00$ a year (6 Vols., 12 issues). Special rate: $\$ 36.00$ a year to individual members of supporting institutions.

Subscriptions, orders for back numbers, and changes of address should be sent to Pacific Journal of Mathematics, 103 Highland Boulevard, Berkeley, California, 94708.

PUBLISHED BY PACIFIC JOURNAL OF MATHEMATICS, A NON-PROFIT CORPORATION

Printed at Kokusai Bunken Insatsusha (International Academic Printing Co., Ltd.), 8-8, 3-chome, Takadanobaba, Shinjuku-ku, Tokyo 160, Japan. 


\section{Pacific Journal of Mathematics \\ Vol. 65, No. $2 \quad$ October, 1976}

Andrew Adler, Weak homomorphisms and invariants: an example .......... 293

Howard Anton and William J. Pervin, Separation axioms and metric-like

functions ............................................. 299

Ron C. Blei, Sidon partitions and p-Sidon sets .................... 307

T. J. Cheatham and J. R. Smith, Regular and semisimple modules ........... 315

Charles Edward Cleaver, Packing spheres in Orlicz spaces .............. 325

Le Baron O. Ferguson and Michael D. Rusk, Korovkin sets for an operator on a

space of continuous functions ............................. 337

Rudolf Fritsch, An approximation theorem for maps into Kan fibrations ....... 347

David Sexton Gilliam, Geometry and the Radon-Nikodym theorem in strict

Mackey convergence spaces .................................

William Hery, Maximal ideals in algebras of topological algebra valued

functions ...................................... 365

Alan Hopenwasser, The radical of a reflexive operator algebra ........... 375

Bruno Kramm, A characterization of Riemann algebras................. 393

Peter K. F. Kuhfittig, Fixed points of locally contractive and nonexpansive

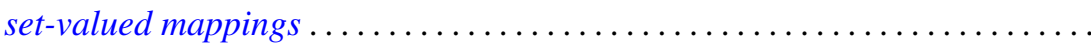

Stephen Allan McGrath, On almost everywhere convergence of Abel means of

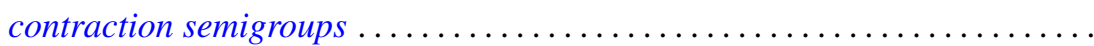

Edward Peter Merkes and Marion Wetzel, A geometric characterization of

indeterminate moment sequences............................ 409

John C. Morgan, II, The absolute Baire property ................... 421

Eli Aaron Passow and John A. Roulier, Negative theorems on generalized convex approximation .................................... 437

Louis Jackson Ratliff, Jr., A theorem on prime divisors of zero and characterizations of unmixed local domains ..............

Ellen Elizabeth Reed, A class of $T_{1}$-compactifications................... 471

Maxwell Alexander Rosenlicht, On Liouville's theory of elementary

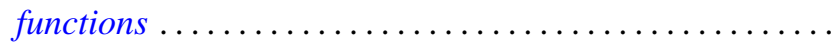

Arthur Argyle Sagle, Power-associative algebras and Riemannian

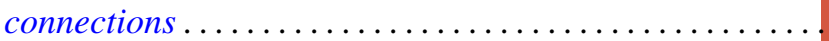

Chester Cornelius Seabury, On extending regular holomorphic maps from Stein manifolds...

Elias Sai Wan Shiu, Commutators and numerical ranges of powers of operators ...................................

Donald Mark Topkis, The structure of sublattices of the product of $n$ lattices ... 525

John Bason Wagoner, Delooping the continuous $K$-theory of a valuation

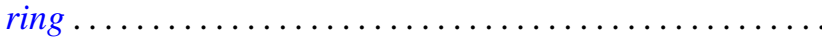

Ronson Joseph Warne, Standard regular semigroups...........

Anthony William Wickstead, The centraliser of $E \otimes_{\lambda} F \ldots$. 